\title{
Propagation in Lossy Rectangular Waveguides
}

\author{
Kim Ho Yeap', Choy Yoong Tham², \\ Ghassan Yassin ${ }^{3}$ and Kee Choon Yeong ${ }^{1}$ \\ 1Tunku Abdul Rahman University \\ 2Wawasan Open University \\ ${ }^{3}$ University of Oxford \\ 1,2 Malaysia \\ ${ }^{3}$ United Kingdom
}

\section{Introduction}

In millimeter and submillimeter radio astronomy, waveguide heterodyne receivers are often used in signal mixing. Wave guiding structures such as circular and rectangular waveguides are widely used in such receiver systems to direct and couple extraterrestrial signals at millimeter and submillimeter wavelengths to a mixer circuit (Carter et al., 2004; Boifot et al., 1990; Withington et al., 2003).

To illustrate in detail the applications of waveguides in receiver systems, a functional block diagram of a typical heterodyne receiver in radio telescopes is shown in Fig. 1 (Chattopadhyay et al., 2002). The electromagnetic signal (RF signal) from the antenna is directed down to the front end of the receiver system via mirrors and beam waveguides (Paine et al., 1994). At the front end of the receiver system, such as the sideband separating receiver designed for the ALMA band 7 cartridge (Vassilev and Belitsky, 2001a; Vassilev and Belitsky, 2001b; Vassilev et al., 2004), the RF signal is channelled from the aperture of the horn through a circular and subsequently a rectangular waveguide, before being coupled to the mixer. In the mixer circuit, a local oscillator (LO) signal which is generally of lower frequency is then mixed with the RF signal, to down convert the RF signal to a lower intermediate frequency (IF) signal. Here, a superconductor-insulator-superconductor (SIS) heterodyne mixer is commonly implemented for the process of down conversion. At the back end of the system, the IF signal goes through multiple stages of amplification and is, eventually, fed to a data analysis system such as an acousto-optic spectrometer. The data analysis system will then be able to perform Fourier transformation and record spectral information about the input signal.

The front-end receiver noise temperature $T_{R}$ is determined by a number of factors. These include the mixer noise temperature $T_{M}$, the conversion loss $C_{\text {Loss, }}$ the noise temperature of the first IF amplifier $T_{I F}$, and the coupling efficiency between the IF port of the junction and the input port of the first IF amplifier $\eta_{I F}$. A comparison of the performance of different SIS waveguide receivers is listed in Table 1 (Walker et al., 1992). It can be seen that the value of $T_{R}$ for the $230 \mathrm{GHz}$ system is a factor of 3 to 4 less than that achieved with the $492 \mathrm{GHz}$ system. The decrease in system performance at $492 \mathrm{GHz}$ is due to the increase of $C_{\text {Loss }}$ and $T_{M}$ by a factor of approximately 3 . 


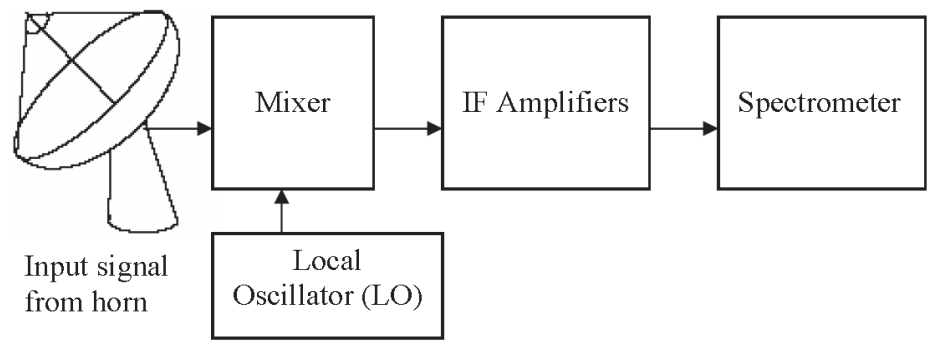

Fig. 1. Block diagram of a heterodyne receiver.

\begin{tabular}{|l|l|l|l|}
\hline SIS Junction & $\mathrm{Nb}$ & $\mathrm{Pb}$ & $\mathrm{Nb}$ \\
\hline Center Frequency $(\mathrm{GHz})$ & 230 & 345 & 492 \\
\hline$T_{R}(\mathrm{~K})$ & 48 & 159 & 176 \\
\hline$T_{M}(\mathrm{~K})$ & 34 & 129 & 123 \\
\hline$C_{\text {Loss }}(\mathrm{dB})$ & 3.1 & 8.1 & 8.9 \\
\hline$T_{I F}(\mathrm{~K})$ & 7.0 & 4.2 & 6.8 \\
\hline
\end{tabular}

Table 1. Comparison of SIS receiver performance.

Since the input power level of the weak millimeter and submillimeter signals is quite small i.e. of the order of $10^{-18}$ to $10^{-20} \mathrm{~W}$ (Shankar, 1986), it is therefore of primary importance to minimize the conversion loss $C_{\text {Loss }}$ of the mixer circuit. One way of doing so, is to ensure that the energy of the LO and, in particular, the RF signals is channelled and coupled from the waveguides to the mixer circuit in a highly efficient manner. It is simply too time consuming and too expensive to develop wave guiding structures in a receiver system on a trial-anderror basis. To minimize the loss of the propagating signals, the availability of an accurate and easy-to-use mathematical model to compute the loss of such signals in wave guiding structures is, of course, central to the development of receiver circuits.

\section{Related work}

Analysis of the propagation of wave in circular cylindrical waveguides has already been widely performed (Glaser, 1969; Yassin et al., 2003; Claricoats, 1960a; Claricoats, 1960b; Chou and Lee, 1988). The analyses by these authors are all based on the rigorous method formulated by Stratton (1941). In Stratton's formulation, the fields at the wall surface are made continuous into the wall material. Assumption made on the field decaying inside the wall material yields relations which allow the propagation constant to be determined. Due to the difficulty in matching the boundary conditions in Cartesian coordinates, this approach, however fails to be implemented in the case of rectangular waveguides. A similar rigorous technique to study the attenuation of rectangular waveguides is not available hitherto.

The perturbation power-loss method has been commonly used in analyzing wave attenuation in lossy (Stratton, 1941; Seida, 2003; Collin 1991; Cheng, 1989) and superconducting (Winters and Rose, 1991; Ma, 1998; Wang et al., 1994; Yalamanchili et al., 1995) rectangular waveguides; respectively. This is partly due to its ability to produce simple analytical solution, and also partly because it gives reasonably accurate result at frequencies $f$ well above its cutoff frequency $f_{c}$. In this method, the field expressions are derived by assuming that the walls to be of infinite conductivity. This allows the solution to 
be separated into pure Transverse Electric (TE) and Transverse Magnetic (TM) modes. For a waveguide with finite conductivity, however, a superposition of both TE and TM modes is necessary to satisfy the boundary conditions (Stratton, 1941; Yassin et al., 2003). This is because, unlike those of the lossless case, the modes in a lossy waveguide are no longer mutually orthogonal to each other (Collin, 1991). To calculate the attenuation, ohmic losses are assumed due to small field penetration into the conductor surface. Results however show that this method fails near cutoff, as the attenuation obtained diverges to infinity when the signal frequency $f$ approaches the cutoff $f_{c}$. Clearly, it is more realistic to expect losses to be high but finite rather than diverging to infinity. The inaccuracy in the powerloss method at cutoff is due to the fact that the field equations are assumed to be identical to those of a lossless waveguide. Since a lossless waveguide behaves exactly like an ideal high pass filter, signals cease to propagate at $f$ below $f_{c}$.

It can be seen that the assumption of lossless fields fail to give an insight or deeper understanding on the mechanism of the propagation of wave in practical lossy waveguides. Moreover, at very high frequency - especially that approaches the millimeter and submillimeter wavelengths - the loss tangent of the conducting wall decreases. Therefore, such assumption turns out to be inaccurate at very high frequency. Although Stratton (1941) has developed a truly fundamental approach to analyze waveguides, his approach is only restricted to the case of circular waveguides and could not be applied to rectangular waveguides. The workhorse of this chapter is, therefore, to develop a novel and accurate formulation - i.e. one that does not assume lossless boundary conditions - to investigate the loss of waves in rectangular waveguides. In particular, the new method shall be found more accurate and useful for waveguides operating at very high frequencies, such as those in the millimeter and submillimeter wavelengths. In Yeap et al. (2009), a simple method to compute the loss of waves in rectangular waveguides has been developed. However, the drawback of the method is that different sets of characteristic equation are required to solve for the propagation constants of different modes. Here, the method proposed in Yeap et al. (2009) shall be developed further so that the loss of different modes can be conveniently computed using only a single set of equation. For convenience purpose, the new method shall be referred to as the boundary-matching method in the subsequent sections.

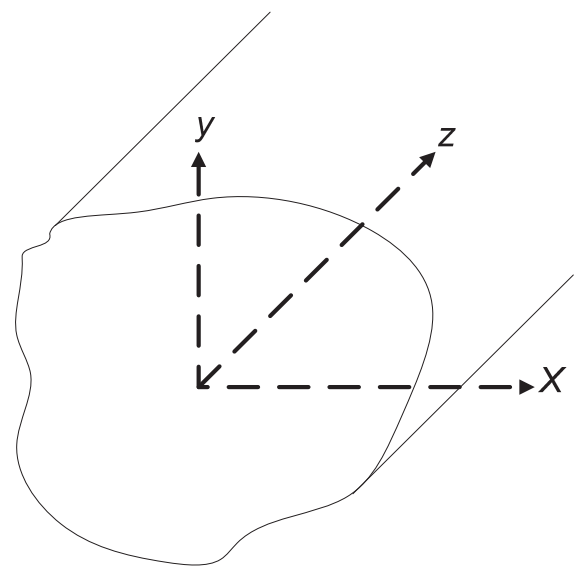

Fig. 2. A waveguide with arbitrary geometry. 


\section{General wave behaviours along uniform guiding structures}

As depicted in Fig. 2, a time harmonic field propagating in the $\mathrm{z}$ direction of a uniform guiding structure with arbitrary geometry can be expressed as a combination of elementary waves having a general functional form (Cheng, 1989)

$$
\psi=\psi^{0}(x, y) \exp \left[\mathrm{j}\left(\omega t+k_{z} z\right)\right]
$$

where $\Psi^{0}(x, y)$ is a two dimensional vector phasor that depends only on the cross-sectional coordinates, $\omega=2 \pi f$ the angular frequency, and $k_{z}$ is the propagation constant.

Hence, in using phasor representation in equations relating field quantities, the partial derivatives with respect to $t$ and $z$ may be replaced by products with $\mathrm{j} \omega$ and $\mathrm{j} k_{z}$, respectively; the common factor $\exp \left[\mathrm{j}\left(\omega t+k_{z} z\right)\right]$ can be dropped. Here, the propagation constant $k_{z}$ is a complex variable, which consists of a phase constant $\beta_{z}$ and an attenuation constant $a_{z}$

$$
k_{z}=\beta_{z}-\mathrm{j} \alpha_{z}
$$

The field intensities in a charge-free dielectric region (such as free-space), satisfy the following homogeneous vector Helmholtz's equation

$$
\nabla^{2} \psi_{z}+\left(k^{2}-k_{z}^{2}\right) \psi_{z}=0
$$

where $\psi_{z}$ is the longitudinal component of $\psi, \nabla^{2}$ is the Laplacian operator for the transverse coordinates, and $k$ is the wavenumber in the material. For waves propagating in a hollow waveguide, $k=k_{0}$, the wavenumber in free-space.

It is convenient to classify propagating waves into three types, in correspond to the existence of the longitudinal electric field $E_{z}$ or longitudinal magnetic $H_{z}$ field:

1. Transverse electromagnetic (TEM) waves. A TEM wave consists of neither electric fields nor magnetic fields in the longitudinal direction.

2. Transverse magnetic (TM) waves. A TM wave consists of a nonzero electric field but zero magnetic field in the longitudinal direction.

3. Transverse electric (TE) waves. A TE wave consists of a zero electric field but nonzero magnetic field in the longitudinal direction.

Single-conductor waveguides, such as a hollow (or dielectric-filled) circular and rectangular waveguide, cannot support TEM waves. This is because according to Ampere's circuital law, the line integral of a magnetic field around any closed loop in a transverse plane must equal the sum of the longitudinal conduction and displacement currents through the loop. However, since a single-conductor waveguide does not have an inner conductor and that the longitudinal electric field is zero, there are no longitudinal conduction and displacement current. Hence, transverse magnetic field of a TEM mode cannot propagate in the waveguide (Cheng, 1989).

\section{Fields in cartesian coordinates}

For waves propagating in a rectangular waveguide, such as that shown in Fig. 3, Helmholtz's equation in (3) can be expanded in Cartesian coordinates to give 


$$
\frac{\partial^{2} \psi_{z}}{\partial x^{2}}+\frac{\partial^{2} \psi_{z}}{\partial y^{2}}+\left(k^{2}-k_{z}^{2}\right) \psi_{z}=0
$$

By applying the method of separation of variables, $\psi_{z}$ can be expressed as

$$
\psi_{z}=X(x) Y(y)
$$

Equation (4) can thus be separated into two sets of second order differential equations, as shown below (Cheng, 1989)

$$
\begin{aligned}
& \frac{d^{2} X(x)}{d x^{2}}+k_{x}{ }^{2} X(x)=0 \\
& \frac{d^{2} Y(y)}{d y^{2}}+k_{y}{ }^{2} Y(y)=0
\end{aligned}
$$

where $k_{x}$ and $k_{y}$ are the transverse wavenumbers in the $x$ and $y$ directions, respectively. The longitudinal fields can be obtained by solving (6) and (7) based on a set of boundary conditions and substituting the solutions into (5).

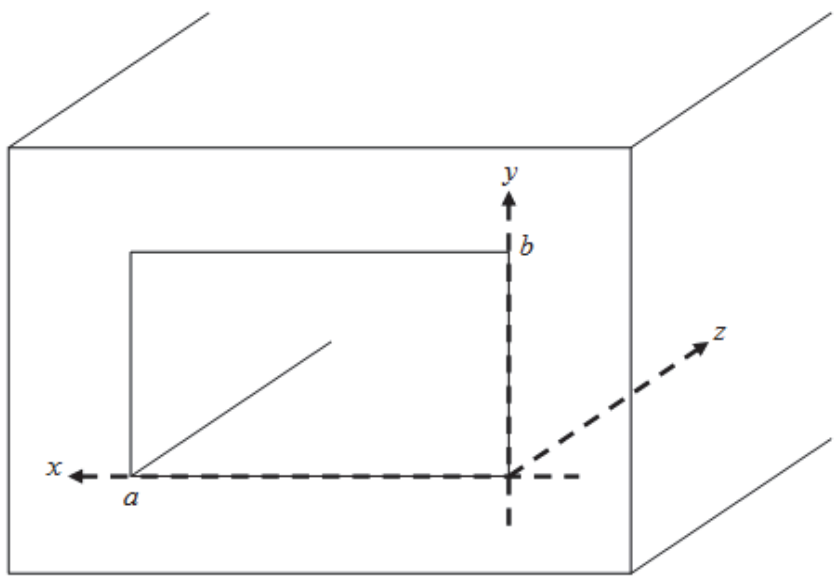

Fig. 3. The cross section of a rectangular waveguide.

The transverse field components can be derived by substituting the longitudinal field components into Maxwell's source free curl equations

$$
\begin{aligned}
& \nabla \times E=-\mathrm{j} \omega \mu H \\
& \nabla \times H=\mathrm{j} \omega \varepsilon E
\end{aligned}
$$

where $\varepsilon$ and $\mu$ are the permittivity and permeability of the material, respectively. Expressing the transverse field components in term of the longitudinal field components $E_{z}$ and $H_{z}$, the following equations can be obtained (Cheng, 1989) 


$$
\begin{gathered}
H_{x}=\frac{-j}{k_{x}^{2}+k_{y}^{2}}\left(k_{z} \frac{d H_{z}}{d x}-\omega \varepsilon \frac{d E_{z}}{d y}\right) \\
H_{y}=\frac{-j}{k_{x}^{2}+k_{y}^{2}}\left(k_{z} \frac{d H_{z}}{d y}+\omega \varepsilon \frac{d E_{z}}{d x}\right) \\
E_{x}=\frac{-j}{k_{x}^{2}+k_{y}^{2}}\left(k_{z} \frac{d E_{z}}{d x}+\omega \mu \frac{d H_{z}}{d y}\right) \\
E_{y}=\frac{-j}{k_{x}^{2}+k_{y}^{2}}\left(k_{z} \frac{d E_{z}}{d y}-\omega \mu \frac{d H_{z}}{d x}\right)
\end{gathered}
$$

\section{Review of the power-loss method}

In the subsequent sections, analysis and comparison between the perturbation power-loss method and the new boundary matching method shall be performed. Hence, in order to present a complete scheme, the derivation of the conventional power-loss method is briefly outlined in this section.

The attenuation of electromagnetic waves in waveguides can be caused by two factors - i.e. the attenuation due to the lossy dielectric material $a_{z(d)}$, and that due to the ohmic losses in imperfectly conducting walls $a_{z(c)}$ (Cheng, 1989)

$$
a_{z}=a_{z(d)}+a_{z(c)},
$$

For a conducting waveguide, the inner core is usually filled with low-loss dielectric material, such as air. Hence, $a_{z(d)}$ in (14) shall be assumed zero in the power-loss method and the loss in a waveguide is assumed to be caused solely by the conduction loss. It could be seen later that such assumption is not necessary in the new boundary-matching method. Indeed, the new method inherently accounts for both kinds of losses in its formulation.

The approximate power-loss method assumes that the fields' expression in a highly but imperfectly conducting waveguide, to be the same as those of a lossless waveguide. Hence, $k_{x}, k_{y}$, and $k_{z}$ are given as (Cheng, 1989)

$$
\begin{aligned}
& k_{x}=\frac{m \pi}{a} \\
& k_{y}=\frac{n \pi}{b} \\
& k_{z}=\beta_{z}
\end{aligned}
$$

where $a$ and $b$ are the width and height, respectively, of the rectangular waveguide; whereas $m$ and $n$ denote the number of half cycle variations in the $x$ and $y$ directions, respectively. Every combination of $m$ and $n$ defines a possible mode for $\mathrm{TE}_{m n}$ and $\mathrm{TM}_{m n}$ waves.

Conduction loss is assumed to occur due to small fields' penetration into the conductor surfaces. According to the law of conservation of energy, the attenuation constant due to conduction loss can be derived as (Cheng, 1989) 


$$
\alpha_{z}=\frac{P_{L}}{2 P_{z}}
$$

where $P_{z}$ is the time-average power flowing through the cross-section and $P_{L}$ the timeaverage power lost per unit length of the waveguide.

Solving for $P_{L}$ and $P_{z}$ based on Poynting's theorem, the attenuation constant $a_{z}$ for TM and TE modes - i.e. $a_{z(\mathrm{TM})}$ and $a_{z(\mathrm{TE})}$, respectively, can thus be expressed as (Collin, 1991)

$$
\begin{gathered}
\alpha_{z(T M)}=\frac{2 R_{s}\left(m^{2} b^{3}+n^{2} a^{3}\right)}{\eta a b \sqrt{1-\left(\frac{f_{c}}{f}\right)^{2}}\left(m^{2} b^{2}+n^{2} a^{2}\right)} \\
\alpha_{z(T E)}=\frac{2 R_{s}}{\eta b \sqrt{1-\left(\frac{f_{c}}{f}\right)^{2}}}\left\{\left(1+\frac{b}{a}\right)\left(\frac{f_{c}}{f}\right)^{2}+\frac{b}{a}\left[1-\left(\frac{f_{c}}{f}\right)^{2}\right]\left[\frac{m^{2} a b+n^{2} a^{2}}{(m b)^{2}+(n a)^{2}}\right]\right\}
\end{gathered}
$$

where $R_{s}$ is the surface resistance, $f_{c}$ the cutoff frequency, and $\eta$ the intrinsic impedance of free space.

\section{The new boundary-matching method}

It is apparent that, in order to derive the approximate power-loss equations illustrated in section 5, the field equations must be assumed to be lossless. In a lossless waveguide, the boundary condition requires that the resultant tangential electric field $E_{t}$ and the normal derivative of the tangential magnetic field $\partial H_{t} / \partial a_{n}$ to vanish at the waveguide wall, where $a_{n}$ is the normal direction to the waveguide wall. In reality, however, this is not exactly the case. The conductivity of a practical waveguide is finite. Hence, both $E_{t}$ and $\partial H_{t} / \partial a_{n}$ are not exactly zero at the boundary of the waveguide. Besides, the loss tangent of a material decreases in direct proportion with the increase of frequency. Hence, a highly conducting wall at low frequency may exhibit the properties of a lossy dielectric at high frequency, resulting in inaccuracy using the assumption at millimeter and submillimeter wavelengths. In order to model the field expressions closer to those in a lossy waveguide and to account for the presence of fields inside the walls, two phase parameters have been introduced in the new method. The phase parameters - i.e $\phi_{x}$ and $\phi_{y}$, are referred to as the field's penetration factors in the $x$ and $y$ directions, respectively. It is worthwhile noting that, with the introduction of the penetration factors, $E_{t}$ and $\partial H_{t} / \partial a_{n}$ do not necessarily decay to zero at the boundary, therefore allowing the effect of not being a perfect conductor at the waveguide wall.

\subsection{Fields in a lossy rectangular waveguide}

For waves propagating in a lossy hollow rectangular waveguide, as shown in Figure 3, a superposition of TM and TE waves is necessary to satisfy the boundary condition at the wall (Stratton, 1941; Yassin et al., 2003). The longitudinal electric and magnetic field components $E_{z}$ and $H_{z}$, respectively, can be derived by solving Helmholtz's homogeneous equation in 
Cartesian coordinate. Using the method of separation of variables (Cheng, 1989), the following set of field equations is obtained

$$
\begin{gathered}
E_{z}=E_{0} \sin \left(k_{x} x+\phi_{x}\right) \sin \left(k_{y} y+\phi_{y}\right) \\
H_{z}=H_{0} \cos \left(k_{x} x+\phi_{x}\right) \cos \left(k_{y} y+\phi_{y}\right)
\end{gathered}
$$

where $E_{0}$ and $H_{0}$ are constant amplitudes of the fields.

The propagation constant $k_{z}$ for each mode will be found by solving for $k_{x}$ and $k_{y}$ and substituting the results into the dispersion relation

$$
k_{z}=\sqrt{k_{0}^{2}-k_{x}^{2}-k_{y}^{2}}
$$

Equations (21) and (22) must also apply to a perfectly conducting waveguide. In that case $E_{z}$ and $\partial H_{z} / \partial a_{n}$ are either at their maximum magnitude or zero at both $x=a / 2$ and $y=b / 2$ i.e. the centre of the waveguide, therefore

$$
\sin \left(\frac{k_{x} a}{2}+\phi_{x}\right)=\sin \left(\frac{k_{y} b}{2}+\phi_{y}\right)= \pm 1 \text { or } 0
$$

Solving (24), the penetration factors are obtained as,

$$
\begin{aligned}
& \phi_{x}=\frac{\left(m \pi-k_{x} a\right)}{2} \\
& \phi_{y}=\frac{\left(n \pi-k_{y} b\right)}{2}
\end{aligned}
$$

For waveguides with perfectly conducting wall, $k_{x}=m \pi / a$ and $k_{y}=n \pi / b$, (25) and (26) result in zero penetration and $E_{z}$ and $H_{z}$ in (21) and (22) are reduced to the fields of a lossless waveguide. To take the finite conductivity into account, $k_{x}$ and $k_{y}$ are allowed to take complex values yielding non-zero penetration of the fields into the waveguide material

$$
\begin{aligned}
& k_{x}=\beta_{x}-j \alpha_{x} \\
& k_{y}=\beta_{y}-j \alpha_{y}
\end{aligned}
$$

where $\beta_{x}$ and $\beta_{y}$ are the phase constants and $a_{x}$ and $a_{y}$ are the attenuation constants in the $x$ and $y$ directions, respectively. Substituting the transverse wavenumbers in (27) and (28) into (23), the propagation constant of the waveguide $k_{z}$ results in a complex value, therefore, yielding loss in wave propagation.

Substituting (21) and (22) into (10) to (13), the fields are obtained as

$$
H_{x}=\frac{j\left[k_{z} k_{x} H_{0}+\omega \varepsilon_{0} k_{y} E_{0}\right] \sin \left(k_{x} x+\varphi_{x}\right) \cos \left(k_{y} y+\phi_{y}\right)}{k_{x}^{2}+k_{y}^{2}}
$$




$$
\begin{aligned}
& H_{y}=\frac{j\left[k_{z} k_{y} H_{0}-\omega \varepsilon_{0} k_{x} E_{0}\right] \cos \left(k_{x} x+\phi_{x}\right) \sin \left(k_{y} y+\phi_{y}\right)}{k_{x}{ }^{2}+k_{y}{ }^{2}} \\
& E_{x}=-\frac{j\left[k_{z} k_{x} E_{0}-\omega \mu_{0} k_{y} H_{0}\right] \cos \left(k_{x} x+\phi_{x}\right) \sin \left(k_{y} y+\phi_{y}\right)}{k_{x}{ }^{2}+k_{y}{ }^{2}} \\
& E_{y}=-\frac{j\left[k_{z} k_{y} E_{0}+\omega \mu_{0} k_{x} H_{0}\right] \sin \left(k_{x} x+\phi_{x}\right) \cos \left(k_{y} y+\phi_{y}\right)}{k_{x}{ }^{2}+k_{y}{ }^{2}}
\end{aligned}
$$

where $\mu_{0}$ and $\varepsilon_{0}$ are the permeability and permittivity of free space, respectively.

\subsection{Formulation}

At the wall, the tangential fields must satisfy the relationship defined by the constitutive properties $\mu_{c}$ and $\varepsilon_{c}$ of the material. The ratio of the tangential component of the electric field to the surface current density at the conductor surface is represented by (Yeap et al., 2009b; Yeap et al., 2010)

$$
\frac{E_{t}}{a_{n} \times H_{t}}=\sqrt{\frac{\mu_{c}}{\varepsilon_{c}}}
$$

where $\mu_{c}$ and $\varepsilon_{c}$ are the permeability and permittivity of the wall material, respectively, and $\sqrt{\frac{\mu_{c}}{\varepsilon_{c}}}$ is the intrinsic impedance of the wall material. The dielectric constant is complex and $\varepsilon_{c}$ may be written as

$$
\varepsilon_{c}=\varepsilon_{0}-j \frac{\sigma_{c}}{\omega}
$$

where $\sigma_{c}$ is the conductivity of the wall.

In order to estimate the loss of waves in millimeter and submillimeter wavelengths more accurately, a more evolved model than the conventional constant conductivity model used at microwave frequencies is necessary. Here, Drude's model is applied for the frequency dependent conductivity $\sigma_{c}$ (Booker, 1982)

$$
\sigma_{c}=\frac{\sigma}{(1+j \omega \tau)}
$$

where $\sigma$ is the conventional constant conductivity of the wall material and $\tau$ the mean free time. For most conductors, such as Copper, the mean free time $\tau$ is in the range of $10^{-13}$ to 10-14 s (Kittel, 1986).

At the width surface of the waveguide, $y=b, E_{z} / H_{x}=-E_{x} / H_{z}=\sqrt{\frac{\mu_{c}}{\varepsilon_{c}}}$. Substituting (21), (22), (29), and (31) into (33), the following relationships are obtained 


$$
\begin{aligned}
& \frac{-E_{x}}{H_{z}}=\frac{j}{k_{x}^{2}+k_{y}^{2}}\left(\frac{E_{0}}{H_{0}} k_{z} k_{x}-\omega \mu_{0} k_{y}\right) \tan \left(k_{y} b+\phi_{y}\right)=\sqrt{\frac{\mu_{c}}{\varepsilon_{c}}} \\
& \frac{H_{x}}{E_{z}}=\frac{j}{k_{x}{ }^{2}+k_{y}{ }^{2}}\left(\frac{H_{0}}{E_{0}} k_{z} k_{x}+\omega \varepsilon_{0} k_{y}\right) \cot \left(k_{y} b+\phi_{y}\right)=\sqrt{\frac{\varepsilon_{c}}{\mu_{c}}}
\end{aligned}
$$

Similarly, at the height surface where $x=a$, we obtain $E_{y} / H_{z}=-E_{z} / H_{y}=\sqrt{\frac{\mu_{c}}{\varepsilon_{c}}}$. Substituting (21), (22), (30), and (32) into (33), the following relationships are obtained

$$
\begin{aligned}
& \frac{E_{y}}{H_{z}}=\frac{-j}{k_{x}^{2}+k_{y}^{2}}\left(\frac{E_{0}}{H_{0}} k_{z} k_{y}+\omega \mu_{0} k_{x}\right) \tan \left(k_{x} a+\phi_{x}\right)=\sqrt{\frac{\mu_{c}}{\varepsilon_{c}}} \\
& \frac{-H_{y}}{E_{z}}=\frac{-j}{k_{x}^{2}+k_{y}^{2}}\left(\frac{H_{0}}{E_{0}} k_{z} k_{y}-\omega \varepsilon_{0} k_{x}\right) \cot \left(k_{x} a+\phi_{x}\right)=\sqrt{\frac{\varepsilon_{c}}{\mu_{c}}}
\end{aligned}
$$

In order to obtain nontrivial solutions for (36) and (37), the determinant of the equations must be zero (Yeap et al., 2009a). By letting the determinant of the coefficients of $E_{0}$ and $H_{0}$ in (36) and (37) vanish the following transcendental equations are obtained

$$
\begin{aligned}
& {\left[\frac{j \omega \mu_{0} k_{y} \tan \left(k_{y} b+\phi_{y}\right)}{k_{x}{ }^{2}+k_{y}{ }^{2}}+\sqrt{\frac{\mu_{c}}{\varepsilon_{c}}}\right]\left[\frac{j \omega \varepsilon_{0} k_{y} \cot \left(k_{y} b+\phi_{y}\right)}{k_{x}{ }^{2}+k_{y}{ }^{2}}-\sqrt{\frac{\varepsilon_{c}}{\mu_{c}}}\right]=\left[\frac{k_{z} k_{x}}{k_{x}{ }^{2}+k_{y}{ }^{2}}\right]^{2}} \\
& {\left[\frac{j \omega \mu_{0} k_{x} \tan \left(k_{x} a+\phi_{x}\right)}{k_{x}{ }^{2}+k_{y}{ }^{2}}+\sqrt{\frac{\mu_{c}}{\varepsilon_{c}}}\right]\left[\frac{j \omega \varepsilon_{0} k_{x} \cot \left(k_{x} a+\phi_{x}\right)}{k_{x}{ }^{2}+k_{y}{ }^{2}}-\sqrt{\frac{\varepsilon_{c}}{\mu_{c}}}\right]=\left[\frac{k_{z} k_{y}}{k_{x}{ }^{2}+k_{y}{ }^{2}}\right]^{2}}
\end{aligned}
$$

Since the dominant $\mathrm{TE}_{10}$ mode has the lowest cutoff frequency among all modes and is the only possible mode propagating alone, it is of engineering importance. In the subsequent sections, comparison and detail analysis of the $\mathrm{TE}_{10}$ mode shall be performed. For the $\mathrm{TE}_{10}$ mode, $m$ and $n$ are set to 1 and 0 , respectively. Substituting $m=1$ and $n=0$ into the penetration factors in (25) and (26), the transcendental equations in (38) for $\mathrm{TE}_{10}$ mode can be simplified to

$$
\begin{gathered}
{\left[\sqrt{\frac{\mu_{c}}{\varepsilon_{c}}}+\frac{j \omega \mu_{0} k_{y} \tan \left(k_{y} \frac{b}{2}\right)}{k_{x}{ }^{2}+k_{y}{ }^{2}}\right]\left[\sqrt{\frac{\varepsilon_{c}}{\mu_{c}}}-\frac{j \omega \varepsilon_{0} k_{y} \cot \left(k_{y} \frac{b}{2}\right)}{k_{x}{ }^{2}+k_{y}{ }^{2}}\right]=\left[\frac{j k_{z} k_{x}}{k_{x}{ }^{2}+k_{y}{ }^{2}}\right]^{2}} \\
{\left[\sqrt{\frac{\mu_{c}}{\varepsilon_{c}}}-\frac{j \omega \mu_{0} k_{x} \cot \left(k_{x} \frac{a}{2}\right)}{k_{x}{ }^{2}+k_{y}{ }^{2}}\right]\left[\sqrt{\frac{\varepsilon_{c}}{\mu_{c}}}+\frac{j \omega \varepsilon_{0} k_{x} \tan \left(k_{x} \frac{a}{2}\right)}{k_{x}{ }^{2}+k_{y}{ }^{2}}\right]=\left[\frac{j k_{z} k_{y}}{k_{x}{ }^{2}+k_{y}{ }^{2}}\right]^{2}}
\end{gathered}
$$


In the above equations, $k_{x}$ and $k_{y}$ are the unknowns and $k_{z}$ can then be obtained from the dispersion relation in (23). A multi root searching algorithm, such as the Powell Hybrid root searching algorithm in a NAG routine, can be used to find the roots of $k_{x}$ and $k_{y}$. The routine requires initial guesses of $k_{x}$ and $k_{y}$ for the search. For good conductors, suitable guess values are clearly those close to the perfect conductor values i.e. $\beta_{x}=m \Pi / a, \beta_{y}=n \Pi / b, a_{x}=a_{y}=0$. Hence, for the $\mathrm{TE}_{10}$ mode, the initial guesses for $k_{x}$ and $k_{y}$ are $\Pi / a$ and 0 respectively.)

\subsection{Experimental setup}

To validate the results, experimental measurements had been carried out. The loss as a function of frequency for a rectangular waveguide was measured using a Vector Network Analyzer (VNA). A $20 \mathrm{~cm}$ copper rectangular waveguide with dimensions of $a=1.30 \mathrm{~cm}$ and $b=0.64 \mathrm{~cm}$ such as that shown in Fig. 4 were used in the measurement.

To minimize noise in the waveguide, a pair of chokes had also been designed and fabricated as shown in Fig. 5. A detail design of the choke drawn using AutoCAD are shown in Fig. 6(a) and Fig. 6(b).

In order to allow the waveguide to be connected to the adapters which are of different sizes, a pair of taper transitions had also been used as shown in Fig. 7. Fig. 8 depicts the complete setup of the experiment where the rectangular waveguide was connected to the VNA via tapers, chokes, coaxial cables, and adapters. Before measurement was carried out, the coaxial cables and waveguide adapters were calibrated to eliminate noise from the two devices. The loss in the waveguide was then observed from the $S_{21}$ or $S_{12}$ parameter of the scattering matrix. The measurement was performed in the frequencies at the vicinity of cutoff.

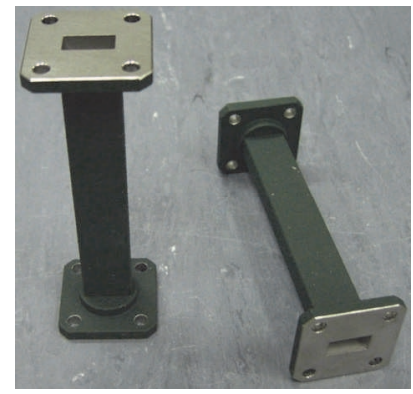

Fig. 4. Rectangular waveguides with width $a=1.30 \mathrm{~cm}$ and height $b=0.64 \mathrm{~cm}$.

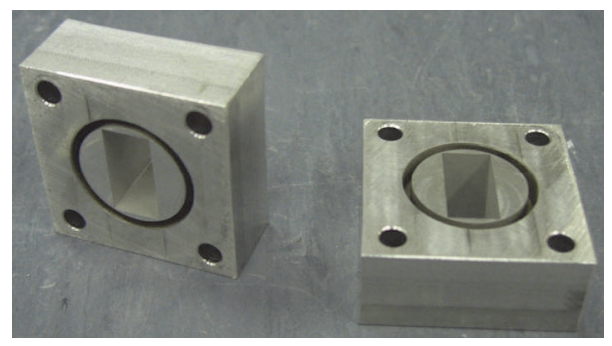

Fig. 5. A pair of chokes made of aluminum. 


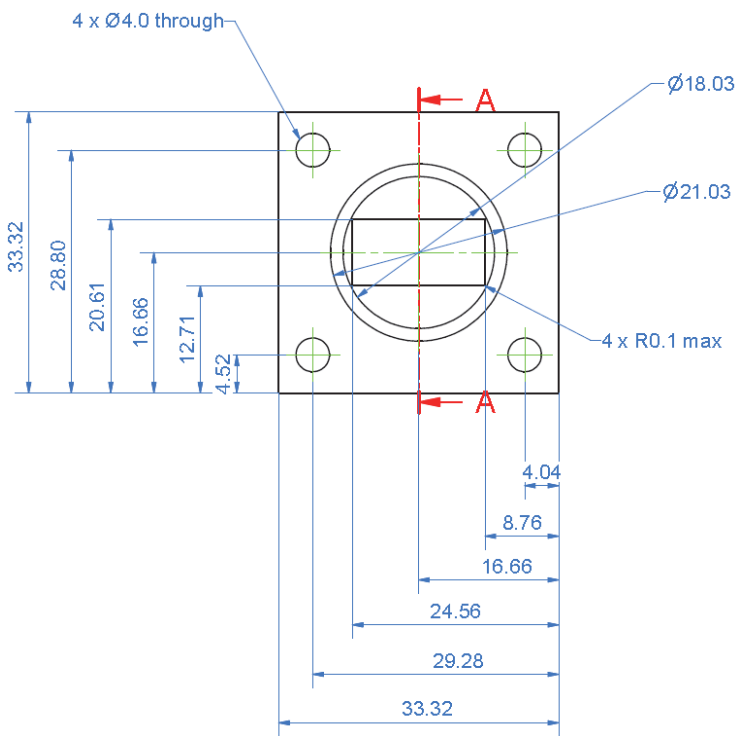

(a)

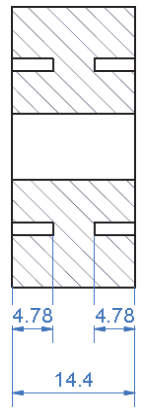

(b)

Fig. 6. Parameters of the (a) cross section and (b) side view of the choke.

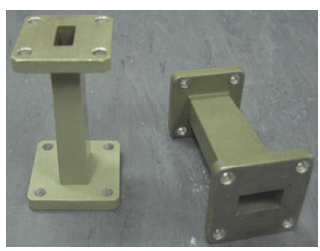

Fig. 7. Taper transitions.

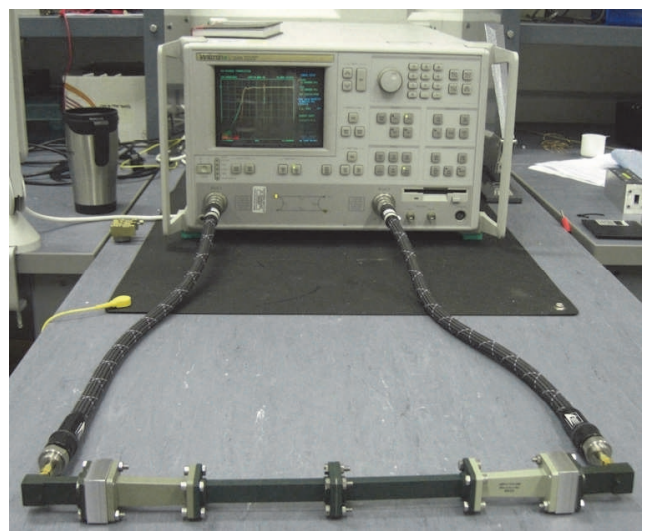

Fig. 8. A $20 \mathrm{~cm}$ rectangular waveguide connected to the VNA, via tapers, chokes, adapters, and coaxial cables. 


\subsection{Results and discussion}

As shown in Fig. 9, a comparison among the attenuation of the $\mathrm{TE}_{10}$ mode near cutoff as computed by the new method, the conventional power-loss method, and the measured $S_{21}$ result was performed. Clearly, the attenuation constant $a_{z}$ computed from the power-loss method diverges sharply to infinity, as the frequency approaches $f_{c}$, and is very different to the simulated results, which show clearly that the loss at frequencies below $f_{c}$ is high but finite. The attenuation computed using the new boundary-matching method, on the other hand, matches very closely with the $S_{21}$ curve, measured using from the VNA. As shown in Table 2, the loss between $11.47025 \mathrm{GHz}$ and $11.49950 \mathrm{GHz}$ computed by the boundarymatching method agrees with measurement to within $5 \%$ which is comparable to the error in the measurement. The inaccuracy in the power-loss method is due to the fact that the fields expressions are assumed to be lossless - i.e. $k_{x}$ and $k_{y}$ are taken as real variables. Analyzing the dispersion relation in (23), it could be seen that, in order to obtain $a_{z}, k_{x}$ and/or $k_{y}$ must be complex, given that the wavenumber in free space is purely real. Although the initial guesses for $k_{x}$ and $k_{y}$ applied in the new boundary-matching method are assumed to be identical with the lossless case, the final results actually converge to complex values when the characteristic equations are solved numerically.

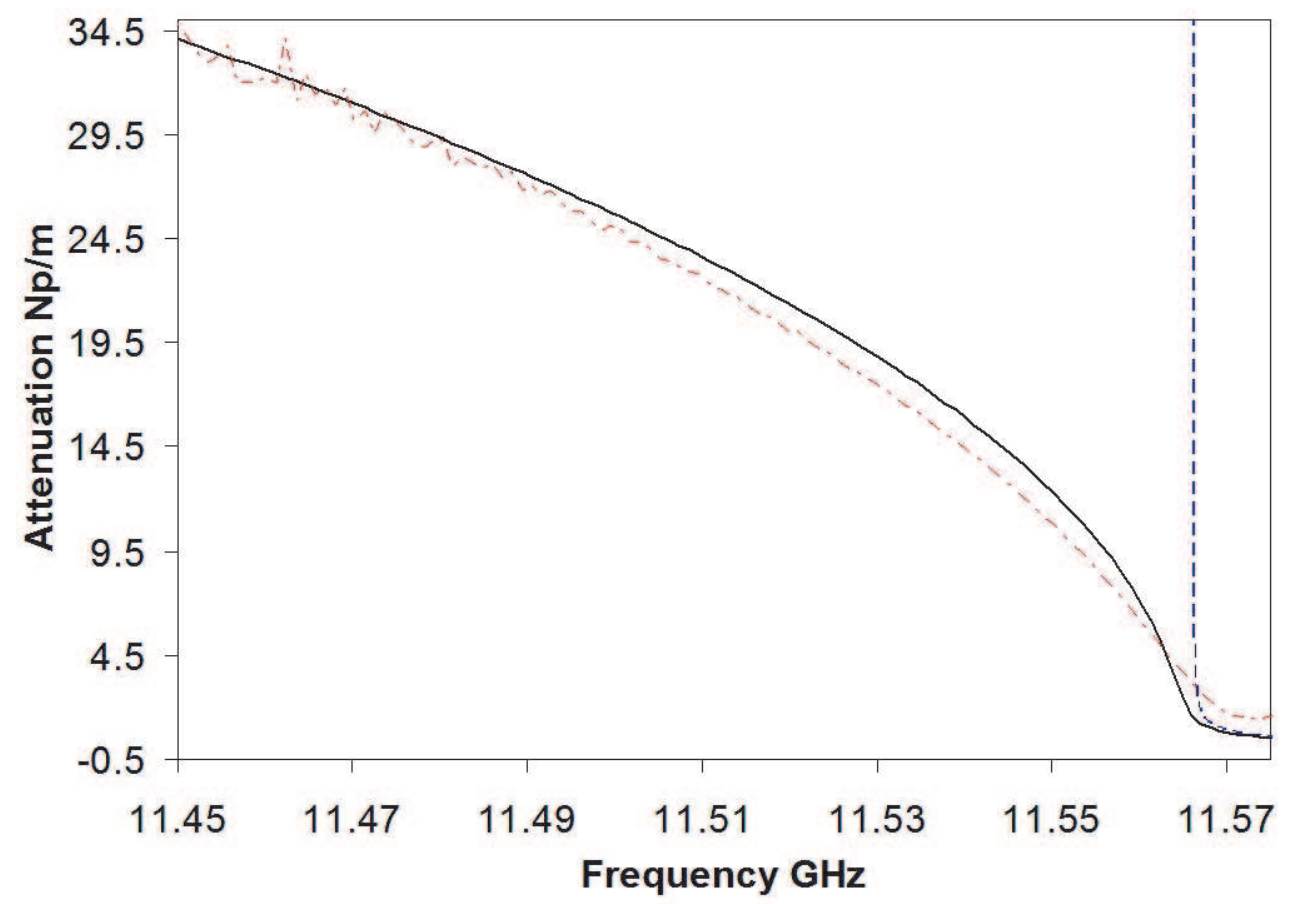

Fig. 9. Attenuation of $\mathrm{TE}_{10}$ mode at the vicinity of cutoff. the new boundary matching method. - - - - - power loss method. -......- S21 measurement.

Fig. 10 shows the attenuation curve when the frequency is extended to higher values. Here, the loss due to $\mathrm{TE}_{10}$ alone could no longer be measured alone, since higher-order modes, such as $\mathrm{TE}_{11}$ and $\mathrm{TM}_{11}$, etc., start to propagate. Close inspection shows that the loss 
predicted by the two methods at higher frequencies is in very close agreement. It is, therefore, sufficed to say that, although the power-loss method fails to predict the attenuation near $f_{c}$ accurately, it is still considered adequate in computing the attenuation of $\mathrm{TE}_{10}$ in lossy waveguides, provided that the frequency $f$ is reasonably above the cutoff $f_{c}$.

As depicted in Fig. 11, at frequencies beyond millimeter wavelengths, however, the loss computed by the boundary-matching method appears to be much higher than those by the power-loss method. The differences can be attributed to the fact that at extremely high frequencies, the loss tangent of the wall material decreases and the field in a lossy waveguide can no longer be approximated to those derived from a perfectly conducting waveguide. At such high frequencies, the wave propagating in the waveguide is a hybrid mode and the presence of the longitudinal electric field $E_{z}$ can no longer be neglected.

\begin{tabular}{|c|c|c|c|}
\hline $\begin{array}{c}\text { Frequency } \\
\text { GHz }\end{array}$ & Experiment & $\begin{array}{c}\text { Boundary-matching } \\
\text { method }\end{array}$ & $\% \Delta$ \\
\hline 11.47025 & 30.17693 & 30.95782 & 2.59 \\
\hline 11.47138 & 30.68101 & 30.77417 & 0.30 \\
\hline 11.47250 & 29.53345 & 30.5894 & 3.58 \\
\hline 11.47363 & 30.51672 & 30.40349 & 0.37 \\
\hline 11.47475 & 30.16449 & 30.21642 & 0.17 \\
\hline 11.47588 & 29.68032 & 30.02816 & 1.17 \\
\hline 11.47700 & 29.09721 & 29.8387 & 2.55 \\
\hline 11.47813 & 28.85077 & 29.648 & 2.76 \\
\hline 11.47925 & 29.25528 & 29.45606 & 0.69 \\
\hline 11.48038 & 29.20923 & 29.26283 & 0.18 \\
\hline 11.48150 & 27.99881 & 29.06831 & 3.82 \\
\hline 11.48263 & 28.38341 & 28.87245 & 1.72 \\
\hline 11.48375 & 28.18551 & 28.67524 & 1.74 \\
\hline 11.48488 & 27.91169 & 28.47664 & 2.02 \\
\hline 11.48600 & 28.08407 & 28.27663 & 0.69 \\
\hline 11.48713 & 27.44495 & 28.07517 & 2.30 \\
\hline 11.48825 & 27.67956 & 27.87224 & 0.70 \\
\hline 11.48938 & 26.84192 & 27.66779 & 3.08 \\
\hline 11.49050 & 26.95767 & 27.46181 & 1.87 \\
\hline 11.49163 & 26.60108 & 27.25425 & 2.46 \\
\hline 11.49275 & 26.78715 & 27.04508 & 0.96 \\
\hline 11.49388 & 26.14928 & 26.83426 & 2.62 \\
\hline 11.49500 & 25.83003 & 26.62174 & 3.07 \\
\hline 11.49613 & 25.82691 & 26.4075 & 2.25 \\
\hline 11.49725 & 25.26994 & 26.19148 & 3.65 \\
\hline 11.49838 & 24.82685 & 25.97365 & 4.62 \\
\hline 11.49950 & 25.1100 & 25.75395 & 2.56 \\
\hline
\end{tabular}

Table 1. Attenuation of $\mathrm{TE}_{10}$ at the vicinity of the cutoff frequency.

Unlike the power-loss method which only gives the value of the attenuation constant, one other advantage of the boundary-matching method is that it is able to account for the phase 


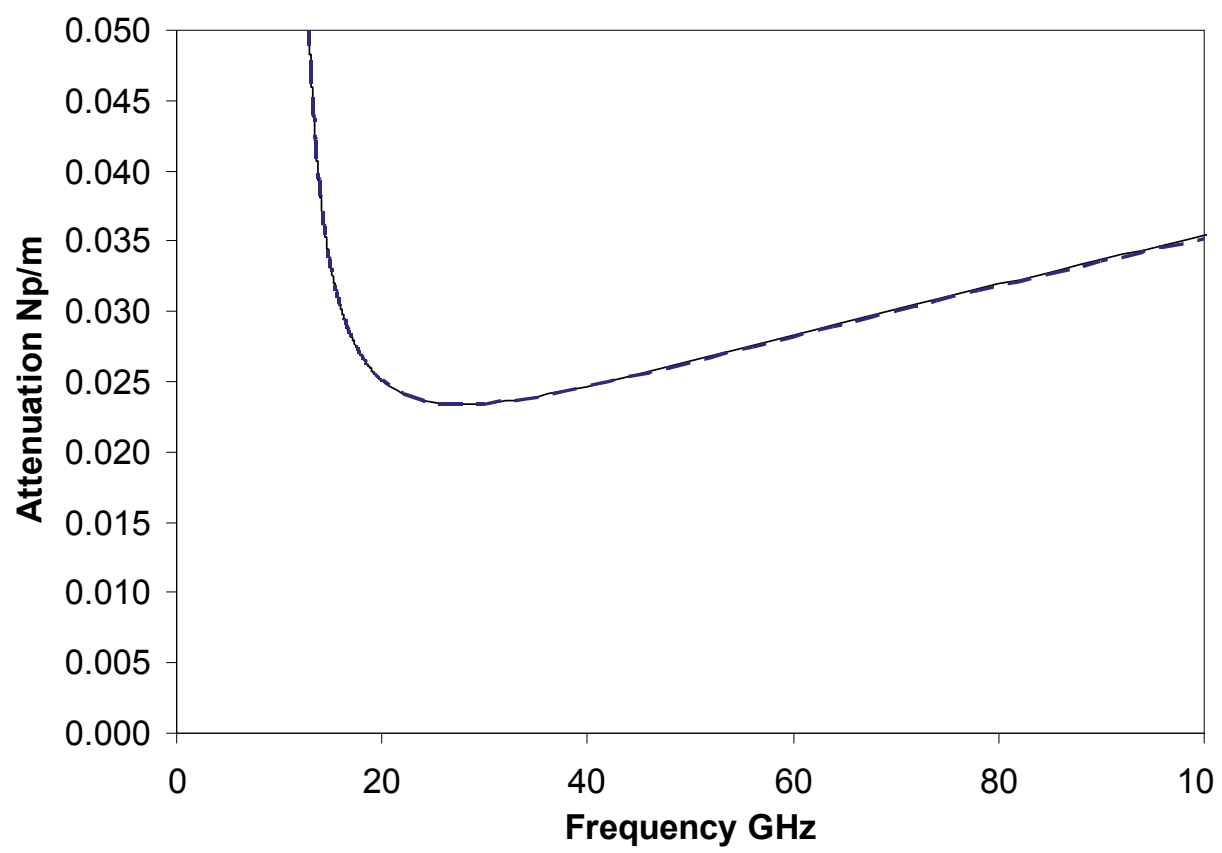

Fig. 10. Attenuation of $\mathrm{TE}_{10}$ mode from 0 to $100 \mathrm{GHz}$. the new boundary matching method. - -.-.--- power loss method.

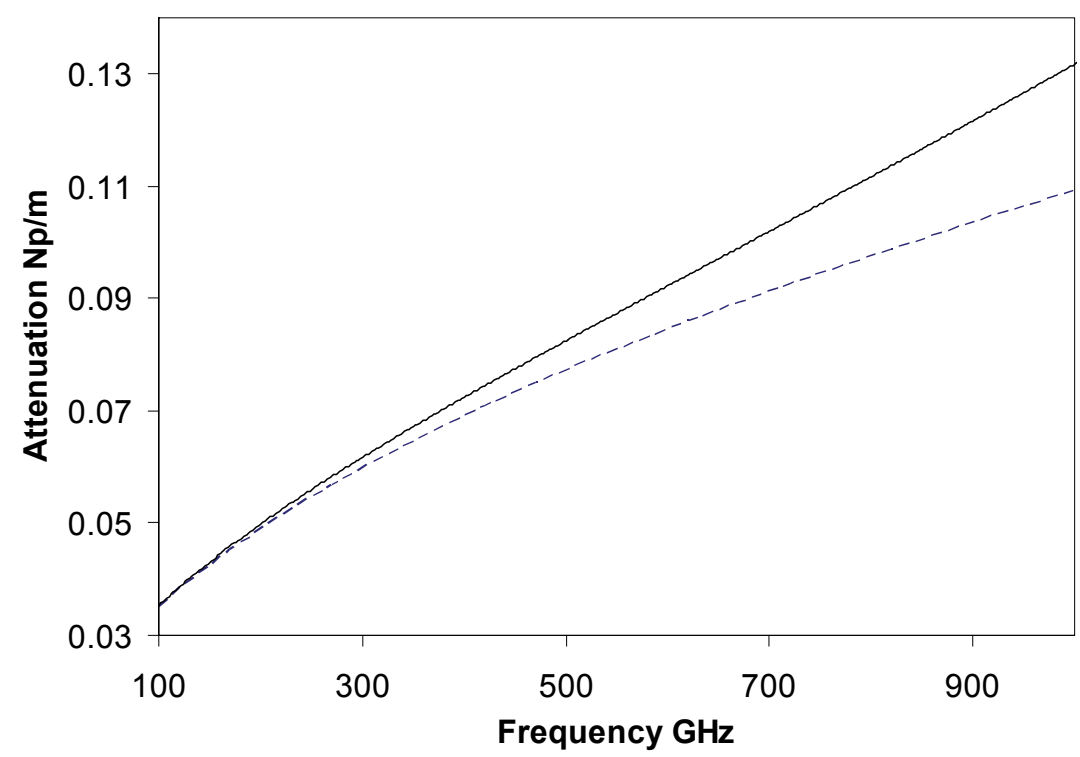

Fig. 11. Attenuation of $\mathrm{TE}_{10}$ mode from $100 \mathrm{GHz}$ to $1 \mathrm{THz}$. the new boundary matching method.---.---- 
constant of the wave as well. A comparison between the attenuation constant and phase constant of a $\mathrm{TE}_{10}$ mode is shown in Fig. 12. As can be observed, as the attenuation in the waveguide gradually decreases, the phase constant increases. Fig. 12 illustrates the change in the mode - i.e. from evanescent below cutoff to propagating mode above cutoff.

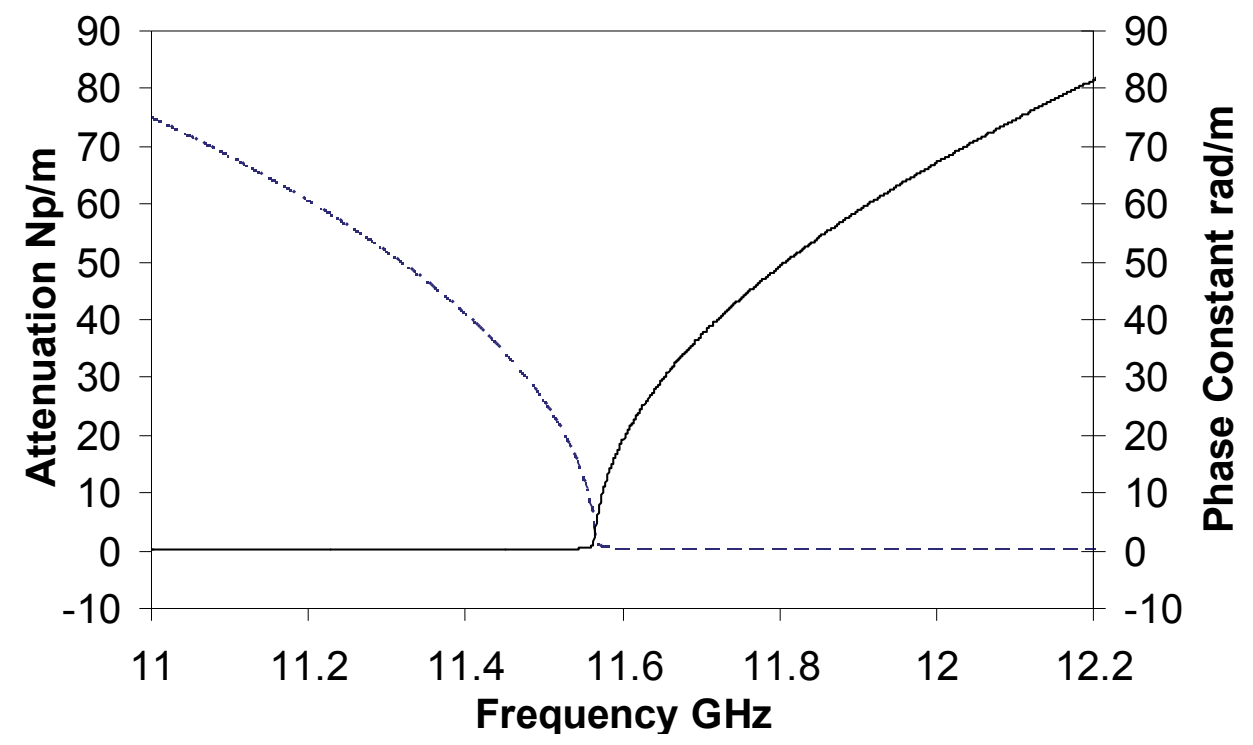

Fig. 12. Propagation constant (phase constant and attenuation constant) of TE10 mode in a lossy rectangular waveguide. constant.

\section{Summary}

A fundamental and accurate technique to compute the propagation constant of waves in a lossy rectangular waveguide is proposed. The formulation is based on matching the fields to the constitutive properties of the material at the boundary. The electromagnetic fields are used in conjunction of the concept of surface impedance to derive transcendental equations, whose roots give values for the wavenumbers in the $x$ and $y$ directions for different TE or TM modes. The wave propagation constant $k_{z}$ could then be obtained from $k_{x}, k_{y}$, and $k_{0}$ using the dispersion relation.

The new boundary-matching method has been validated by comparing the attenuation of the dominant mode with the S21 measurement, as well as, that obtained from the powerloss method. The attenuation curve plotted using the new method matches with the powerloss method at a reasonable range of frequencies above the cutoff. There are however two regions where both curves are found to differ significantly. At frequencies below the cutoff $f_{c}$, the power-loss method diverges to infinity with a singularity at frequency $f=f_{c}$. The new method, however, shows that the signal increases to a highly attenuating mode as the frequencies drop below $f_{c}$. Indeed, such result agrees very closely with the measurement result, therefore, verifying the validity of the new method. At frequencies above $100 \mathrm{GHz}$, the attenuation obtained using the new method increases beyond that predicted by the power-loss method. At $f$ above the millimeter wavelengths, the field in a lossy waveguide 
can no longer be approximated to those of the lossless case. The additional loss predicted by the new boundary-matching method is attributed to the presence of the longitudinal $E_{z}$ component in hybrid modes.

\section{Acknowledgment}

K. H. Yeap acknowledges Boon Kok, Paul Grimes, and Jamie Leech for their advise and discussion.

\section{References}

Boifot, A. M.; Lier, E. \& Schaug-Petersen, T. (1990). Simple and broadband orthomode transducer, Proceedings of IEE, 137, pp. $396-400$

Booker, H. (1982). Energy in Electromagnetism. 1st Edition. Peter Peregrinus.

Carter, M. C.; Baryshev, A.; Harman, M.; Lazareff, B.; Lamb, J.; Navarro, S.; John, D.; Fontana, A. -L.; Ediss, G.; Tham, C. Y.; Withington, S.; Tercero, F.; Nesti, R.; Tan, G. -H.; Sekimoto, Y.; Matsunaga, M.; Ogawa, H. \& Claude, S. (2004). ALMA front-end optics. Proceedings of the Society of Photo Optical Instrumentation Engineers, 5489, pp. $1074-1084$.

Chattopadhyay, G.; Schlecht, E.; Maiwald, F.; Dengler, R. J.; Pearson, J. C. \& Mehdi, I. (2002). Frequency multiplier response to spurious signals and its effects on local oscillator systems in millimeter and submillimeter wavelengths. Proceedings of the Society of Photo-Optical Instrumentation Engineers, 4855, pp. 480 - 488.

Cheng, D. K. (1989). Field and Wave Electromagnetics, Addison Wesley, ISBN 0201528207, US.

Chou, R. C. \& Lee, S. W. (1988). Modal attenuation in multilayered coated waveguides. IEEE Transactions on Microwave Theory and Techniques, 36, pp. 1167 - 1176.

Claricoats, P. J. B. (1960a). Propagation along unbounded and bounded dielectric rods: Part 1. Propagation along an unbounded dielectric rod. IEE Monograph, 409E, pp. $170-$ 176.

Claricoats, P. J. B. (1960b). Propagation along unbounded and bounded dielectric rods: Part 2. Propagation along a dielectric rod contained in a circular waveguide. IEE Monograph, 409E, pp. 177 - 185.

Collin, R. E. (1991). Field Theory of Guided Waves, John Wiley \& Sons, ISBN 0879422378, New York.

Glaser, J. I. (1969). Attenuation and guidance of modes on hollow dielectric waveguides. IEEE Transactions on Microwave Theory and Techniques (Correspondence), 17, pp. 173 176.

Kittel, C. (1986). Introduction to Solid State Physics, John Wiley \& Sons, New York.

Ma, J. (1998). TM-properties of HTS's rectangular waveguides with Meissner boundary condition. International Journal of Infrared and Millimeter Waves, 19, pp. 399 - 408.

Paine, S.; Papa, D. C.; Leombruno, R. L.; Zhang, X. \& Blundell, R. (1994). Beam waveguide and receiver optics for the SMA. Proceedings of the 5th International Symposium on Space Terahertz Technology, University of Michigan, Ann Arbor, Michigan.

Seida, O. M. A. (2003). Propagation of electromagnetic waves in a rectangular tunnel. Applied Mathematics and Computation, 136, pp. 405 - 413.

Shankar, N. U. (1986). Application of digital techniques to radio astronomy measurements, Ph.D. Thesis. Raman Research Institute. Bangalore University. 
Stratton, J. A. (1941). Electromagnetic Theory, McGraw-Hill, ISBN 070621500, New York.

Vassilev, V. \& Belitsky, V. (2001a). A new 3-dB power divider for millimetre-wavelengths. IEEE Microwave and Wireless Components Letters, 11, pp. $30-32$.

Vassilev, V. \& Belitsky, V. (2001b). Design of sideband separation SIS mixer for $3 \mathrm{~mm}$ band. Proceedings of the 12th International Symposium on Space Terahertz Technology, Shelter Island, San Diego, California.

Vassilev, V.; Belitsky, V.; Risacher, C.; Lapkin, I.; Pavolotsky, A. \& Sundin, E. (2004). Design and characterization of a sideband separating SIS mixer for $85-115 \mathrm{GHz}$. Proceedings of the 15th International Symposium on Space Terahertz Technology, Hotel Northampton, Northampton, Masachusetts.

Walker, C. K.; Kooi, J. W.; Chan, M.; Leduc, H. G.; Schaffer, P. L.; Carlstrom, J. E. \& Phillips, T. G. (1992). A low-noise $492 \mathrm{GHz}$ SIS waveguide receiver. International Journal of Infrared and Millimeter Waves, 13, pp. 785 - 798.

Wang, Y.; Qiu, Z. A. \& Yalamanchili, R. (1994). Meissner model of superconducting rectangular waveguides. International Journal of Electronics, 76, pp. 1151 - 1171.

Winters, J. H. \& Rose, C. (1991). High- $T_{c}$ superconductors waveguides: Theory and applications. IEEE Transactions on Microwave Theory and Techniques, 39, pp. 617 623.

Withington, S. (2003). Terahertz astronomical telescopes and instrumentation. Philosophical Transactions of the Royal Society of London, 362, pp. $395-402$.

Yalamanchili, R., Qiu, Z. A., Wang, Y. (1995). Rectangular waveguides with two conventional and two superconducting walls. International Journal of Electronics, 78, pp. $715-727$.

Yassin, G., Tham, C. Y. \& Withington, S. (2003). Propagation in lossy and superconducting cylindrical waveguides. Proceedings of the 14th International Symposium on Space Terahertz Technology, Tucson, Az.

Yeap, K. H., Tham, C. Y., and Yeong, K. C. (2009a). Attenuation of the dominant mode in a lossy rectangular waveguide. Proceedings of the IEEE 9th Malaysia International Conference on Communications, KL., Malaysia.

Yeap, K. H., Tham, C. Y., Yeong, K. C. \& Lim, E. H. (2010). Full wave analysis of normal and superconducting microstrip transmission lines. Frequenz Journal of RF-Engineering and Telecommunications, 64, pp. $59-66$.

Yeap, K. H., Tham, C. Y., Yeong, K. C. \& Yeap, K. H. (2009b). A simple method for calculating attenuation in waveguides. Frequenz Journal of RF-Engineering and Telecommunications, 63, pp. $236-240$. 


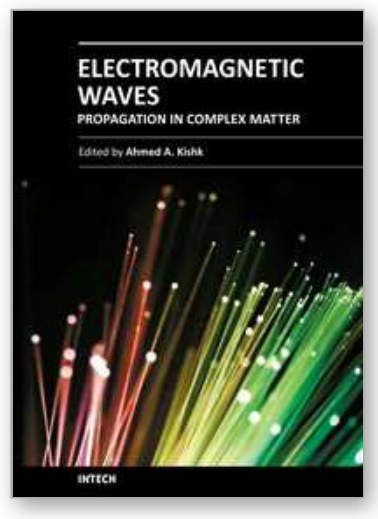

\author{
Electromagnetic Waves Propagation in Complex Matter \\ Edited by Prof. Ahmed Kishk
}

ISBN 978-953-307-445-0

Hard cover, 292 pages

Publisher InTech

Published online 24, June, 2011

Published in print edition June, 2011

This volume is based on the contributions of several authors in electromagnetic waves propagations. Several issues are considered. The contents of most of the chapters are highlighting non classic presentation of wave propagation and interaction with matters. This volume bridges the gap between physics and engineering in these issues. Each chapter keeps the author notation that the reader should be aware of as he reads from chapter to the other.

\title{
How to reference
}

In order to correctly reference this scholarly work, feel free to copy and paste the following:

Kim Ho Yeap, Choy Yoong Tham, Ghassan Yassin and Kee Choon Yeong (2011). Propagation in Lossy Rectangular Waveguides, Electromagnetic Waves Propagation in Complex Matter, Prof. Ahmed Kishk (Ed.), ISBN: 978-953-307-445-0, InTech, Available from: http://www.intechopen.com/books/electromagnetic-wavespropagation-in-complex-matter/propagation-in-lossy-rectangular-waveguides

\section{INTECH}

open science | open minds

\section{InTech Europe}

University Campus STeP Ri

Slavka Krautzeka 83/A

51000 Rijeka, Croatia

Phone: +385 (51) 770447

Fax: +385 (51) 686166

www.intechopen.com

\section{InTech China}

Unit 405, Office Block, Hotel Equatorial Shanghai

No.65, Yan An Road (West), Shanghai, 200040, China

中国上海市延安西路65号上海国际贵都大饭店办公楼 405 单元

Phone: +86-21-62489820

Fax: $+86-21-62489821$ 
(C) 2011 The Author(s). Licensee IntechOpen. This chapter is distributed under the terms of the Creative Commons Attribution-NonCommercialShareAlike-3.0 License, which permits use, distribution and reproduction for non-commercial purposes, provided the original is properly cited and derivative works building on this content are distributed under the same license. 